expression of $\beta 7 \& C L A$ surface homing markers and cytokine content of T-cells (TNF $\alpha$, TGF $\beta$, IFN $\gamma$, IL-10.15.17) was quantified by flow cytometry on stimulated T-cells. Unparied t-test and one-way ANOVA statistical analyses were applied

Results In basal conditions, LDC from HC \& CD patients did not differ in their stimulatory capacity for allogeneic T-cells or in the cytokine profile acquired by T-cells. However, T-cells stimulated by $\mathrm{LDC}$ from $\mathrm{CD}$ patients decreased $\beta 7$ intensity ratio. Following culture with IFX, LDC decreased their stimulatory capacity in a dose-dependent, stepwise fashion in both HC and CD. Culture with IFX did not have any effect on the acquired homing profile of stimulated T-cells, although these T-cells had a trend (not statistically significant) towards lower TNF $\alpha$ and higher IL-17 production

Conclusion The marked reduction in the ability of LDC to stimulate T-cells following culture with IFX represents one plausible explanation for the efficacy of anti TNF-alpha therapies in the treatment of CD. This effect was dose-dependent (within our range of test concentrations) suggesting that higher doses of IFX further reduce T-cell stimulation and may provide one explanation of the clinical benefits of dose escalation in refractory CD

Disclosure of Interest None Declared.

\section{PTH-106 UNRAVELLING THE MECHANISM OF ACTION OF INFLIXIMAB IN CROHN'S DISEASE AND HEALTHY CONTROLS FOLLOWING IN VITRO CULTURE WITH BLOOD- ENRICHED DENDRITIC CELLS}

doi:10.1136/gutjnl-2013-304907.593

${ }^{1, *} \mathrm{~S}$ T C Peake, ${ }^{2} \mathrm{D}$ Bernardo, ${ }^{2} \mathrm{E}$ Mann, ${ }^{1} \mathrm{~J}$ Landy, ${ }^{2} \mathrm{H}$ Omar, ${ }^{2} \mathrm{~S}$ C Knight, ${ }^{1} \mathrm{~A}$ L Hart. ${ }^{1} / \mathrm{BD}$ Unit, St Mark's Hospital; ${ }^{2} A P R G$, Imperial College, London, UK

Introduction Dendritic cells (DC) play a key role in discriminating between commensal microorganisms and harmful pathogens. DC phenotype and cytokine production determine the type of immune response elicited by T-cells following antigen presentation. DC also direct the T-cells to target tissues to perform their function via imprinting tissue-specific homing markers. In $C D$, dysregulation of the immune response to gut microbiota \& aberrant immune cell trafficking play a role in disease pathogenesis. Infliximab (IFX) is an effective treatment for CD but its mechanism of action is unclear. In this study we investigated the in-vitro effect of IFX on phenotype \& ongoing cytokine production of human blood-enriched DC from patients with active CD and healthy controls (HC)

Methods Low density cells (LDC), enriched for DC, were obtained following Ficoll \& Nycoprep separation of blood from patients with active CD (CDAI > 220) and HC. LDC were cultured $\left(0.5 \times 10^{6}\right.$ cells/ $\mathrm{ml})$ with IFX $(1.10 \& 100 \mu \mathrm{g} / \mathrm{ml} \&$ basal $)$ for $24 \mathrm{hr}$. Activation marker (CD40, CD80, HLADR), TLR receptor (TLR2.4) and homing marker (CCR4 5.7.8.9.10, $\beta 7$ ) expression was quantified by flow cytometry. Natural ongoing intracellular cytokine production (TNF $\alpha$, TGF $\beta$ \&IL-6.10.12.15) was assessed via intracellular staining and flow cytometry. Cytokine secretion was measured on cell-free culture supernatants via Multiplex. Unpaired t-test and one-way ANOVA statistical analyses were applied

Results TNF $\alpha$ and IL- 6 were increased in culture supernatants from $\mathrm{CD}$ although their intracellular ongoing cytokine production was decreased. LDC from CD had decreased $\beta 7$ (gut-homing integrin) expression. Following IFX culture, LDC decreased $\beta 7$ expression \& CCR9 intensity ratio (dose-dependent). There was a trend towards reduction in TLR2 and 4 expression (not statistically significant). IL-12 production by LDC from HC was increased following IFX culture. There was a marked reduction in TNF $\alpha$ and IL-12 in cell supernatant following culture with IFX
Conclusion Increased TNF $\alpha$ and IL- 6 in culture supernatants from $\mathrm{CD}$ patients coupled with a decrease in ongoing production by DC suggests a negative cytokine feedback system. Reduced $\beta 7$ expression on LDC from CD patients may suggest that DC have already been recruited to the site of mucosal inflammation. Further experiments are needed to confirm this. Reduced expression of CCR9 and elevated production of IL-12 in LDC cultured with IFX, shows reduced affinity for gut-homing and increased immunogenicity and may suggest a possible mechanism for IFX-induced paradoxical inflammation. The most dramatic effect of IFX was a reduction in TNF $\alpha$ in the supernatant, which is likely to represent neutralisation of the cytokine

Disclosure of Interest None Declared.

\section{PTH-107 KNOWLEDGE OF SOUTH ASIAN POPULATION IN INFLAMMATORY BOWEL DISEASE: CCKNOW- HOW MUCH DO THEY KNOW?}

doi:10.1136/gutjnl-2013-304907.594

1.'S Soteriadou, 'S Hickman, 'J K Limdi. 'Gastroenterology, Pennine Acute NHS Trust, Manchester, UK

Introduction Despite an unprecedented explosion in information and technology and improved understanding of the aetiopathogenesis of Inflammatory bowel diseases (IBD) it remains unclear whether this has translated into a meaningful improvement in patients understanding of their disease. Less is known about South Asian patients with IBD. Our aim was to assess the understanding amongst South Asian patient's using the validated CCKNOW questionnaire ${ }^{1}$.

Methods We conducted a prospective questionnaire-based study of 108 South Asian IBD patients at our institution. Patient demographics, first language, country of origin, educational level and disease duration were recorded. A previously validated 24-item Crohn's and Colitis Knowledge (CCKNOW) questionnaire was used to assess IBD-specific knowledge relating to disease ${ }^{1}$.

Results Forty-three of 108 surveys sent out were returned (39.8\%).

Twenty-three of the 43 patients surveyed were male (53.5\%). Fifteen were born in the UK (34.8\%). English was recorded as the first language in 15 (34.9\%). The highest education level was high school comprehensive in 19 (44.1\%), sixth-form/technical college in 5 $(11.6 \%)$, university in $14(32.6 \%)$, and postgraduate in $3(6.98 \%)$. Fifty one percent of patients described their health to be average or below average, as opposed to good.

Both the mean and median CCKNOW score were 6.74 (Range $0-20)$. Mean scores related to anatomy were 1.21 ( $\mathrm{n}=5 ; 24.1 \%)$, aetiology $0.77(n=2 ; 38.35 \%)$, symptoms $1.55(n=3 ; 38.7 \%)$, diagnosis $0.19(\mathrm{n}=1 ; 18.6 \%)$, treatment and related complications 1.91 $(\mathrm{n}=7 ; 23.6 \%)$, surgery $0.07(\mathrm{n}=1,7 \%)$, complications $0.93(\mathrm{n}=4$; $23.2 \%)$, and pregnancy and fertility related questions $0.12(\mathrm{n}=1$; $11.6 \%)$

Conclusion The knowledge base of South Asian IBD patients was thus deemed poor. More concerning were specific areas of deficient knowledge such as pregnancy and fertility, cancer and treatment related complications with the potential for serious consequences.

Patient education is a key determinant of clinician -patient relationship and an important outcome modifier in chronic disease. IBD teams must guide information needs of specific patient groups through well designed material to enhance knowledge and understanding of disease ultimately impacting on quality of life, treatment adherence and reduction in the use of healthcare resources

Disclosure of Interest None Declared.

\section{REFERENCE}

1. Eaden JA, Abrams K, Mayberry JF. The Crohn's and Colitis Knowledge Score: a test for measuring patient knowledge in inflammatory bowel disease. Am J Gastroenterol. 1999 Dec; 94(12):3560-6. 